\title{
Influence of GENE-X' promotor methylation on the susceptibility to develop PTSD
}

\author{
Renee Simons \\ Maastricht University \\ renee.simons@student.maastrichtuniversity.nl
}

\author{
Laurence de Nijs \\ Maastricht University \\ I.denijs@maastrichtuniverity.nl
}

\section{Astract}

Traumatic events are a prevalent and high risk factor to develop psychiatric disorder like post traumatic stress disorder (PTSD). However, people differ strictly in the susceptibility or resilience to develop such disorders. The exact underlying mechanisms are unknown, but epigenetic mechanisms, such as DNA methylation might play a major role. This was demonstrated by a recent study from our group, who showed significant hypomethylation of GENE-X in the blood of subjects with PTSD. To study brain methylation patterns, the protein expression in PTSD related brain areas of social defeat (SD) mice was compared to control mice (CTR). Expression was visualized by immunohistochemistry (biotin, ABC-kit, $D A B$ ), on human tissue immunofluorescence (biotin, streptavidin) was conducted too. The GENE-X antibody was demonstrated to be specific, since the signal was reduced when performing a pre-incubation with the blocking peptide. Double labelled fluorescence showed GENE-X expression in human temporal neocortex in the cytoplasm and nucleus of neurons, and to a lower extend in astrocytic cytoplasm The staining on SD and CTR mice tissue gave no results about expression sites. This problem was expected to be caused by incorrect fixation of the brain tissue. To conclude, indirect immunohistochemistry allowed visualization of specific GENE-X expression in the human brain. However, the research on the human tissue is not related to stress. Therefore, further research on SD versus CTR material is needed, to prove a causal link between GENE-X hypomethylation in the brain and increased susceptibility to develop PTSD.

1 GENE-X is an alias for the gene that is involved in this research, however, since the previous research is not published yet, the data is still confidential. 
Keywords

Epigenetics, methylation, PTSD, immunohistochemisty, social defeat.

\section{Introduction}

A previous study from our laboratory showed interesting results about the DNA methylation status of GENE-X in relation to the development of a post-traumatic stress disorder (PTSD). For this experiment, researchers studied 96 Dutch and American soldiers who were deployed to Afghanistan or Iraq. Blood samples were collected before and 6 months after the deployment and used to study the genome wide DNA methylation changes. GENE-X's involvement in the pathology of PTSD was proven by hypomethylation of the promotor in the blood of soldiers who developed PTSD after exposure to combat trauma, when compared to soldiers who were resilient to develop PTSD (Unpublished confidential data).

DNA methylation is an epigenetic mechanism that can change the protein expression without changing the DNA sequence. When the promotor of a gene is methylated, the chromatin structure is very solid and this blocks the accessibility of the required transcription factors. The hypomethylation of GENE-X, as seen in soldiers with PTSD, therefore leads to an increased protein expression, this implied GENE-X expression to be involved in the susceptibility to develop PTSD after trauma exposure (1).

Very little is known about the function of this gene in the brain in relation to health and PTSD. We hypothesize prominent GENE-X protein expression in the brain, which differs between PTSD versus controls subjects, especially in brain regions related to PTSD, e.g. the hippocampus, prefrontal cortex and amygdala. Therefore, the aim of the current study will be to investigate the expression pattern of GENE-X in the brain of control mice and in a mouse model of stress (social defeat), as well as in the human brain. This will be performed by the use of immunohistochemical and immunofluorescent stainings to locate the expression of the protein.

\section{Material and methods}

\section{Social defeat model}

The social defeat (SD) model consisted of C58BL6 male mice, that were randomly assigned to an experimental- or control group. The experimental mice were exposed to a dominant mice, while the control (CTR) mice were exposed to another C56BL6 mice without antagonistic behavior. After a confrontation of 10 minutes, the two mice were 
separated using a transparent and perforated wall, and left in their cage for 24 hours. This procedure of social and physical defeat was repeated for 15 consecutive days. At the end of the experiment the mice were sacrificed. In a pilot study of 25 animals, the tissue was perfused with $4 \%$ parafolmaldehyde. In the batch study involving 75 animals, half of mouse brains were immerged-fixated in $4 \%$ paraformaldehyde for one week at $4^{\circ} \mathrm{C}$ and the other halves were fresh frozen.

\section{Immunohistochemistry and immunofluorescence}

Immunohistochemistry on mouse brain was accomplished on free floating sections The first step involved washing of the tissue using Tris-buffered saline (TBS) (Tris 6.o gr/l, $\mathrm{NaCl}$ $8.8 \mathrm{gr} / \mathrm{l}) \mathrm{pH}$ 7.6. Endogenous peroxides were blocked by the incubation with $0.3 \% \mathrm{H}_{2} \mathrm{O}_{2}$, followed by washing with TBS-Triton $0.3 \%$ (TBS-T) pH 7.6, TBS, and TBS-T. Afterwards the tissue was blocked with $2.5 \%$ Bovine Serum Albumin (BSA). After an overnight incubation at $4^{\circ} \mathrm{C}$ with primary antibody (rabbit anti-GENE-X, (1:250 LSBio); rabbit anti-GFAP (1:500, DAKO)), tissues were washed again (TBS-T, TBS, TBS-T). Secondary antibody was added (donkey anti-rabbit biotin labeled, 1:800, Jackson ImmunoResearch) and incubation was followed by washing steps. This was pursued by incubation with Vectastain $A B C$ kit (Vector Laboratories (1:800). Two washing steps with TBS and one with Tris- $\mathrm{HCl} 0.05 \mathrm{M} \mathrm{pH} 7.6$ were performed next. Sections were incubated exactly $10 \mathrm{~min}$ with DAB-Peroxidase substrate solution (0.05\% DAB, $0.015 \% \mathrm{H}_{2} \mathrm{O} 2$, Tris- $\mathrm{HCl} 0.025 \mathrm{M}, \mathrm{pH}$ 7.6) then washed with TBS. Tissue sections were mounted on gelatine coated slides, and let air dried overnight. Slides were dehydrated by consecutively placing them in 50\%,70\%, 96\%, 99,5\% ethanol and ultraclear. Tissues were coverslipped using pertex mounting medium (Histolab). Human tissue was stained on slide and therefore needed to be deparaffinised and re-hydrated using xylene and ethanol concentrations of $99,5 \%$ to $50 \%$. A retrieval step was performed in $0.01 \mathrm{M}$ citrate buffer $\mathrm{pH} 6.0$ in a preheated water bath at $80^{\circ} \mathrm{C}$. The rest of the protocol was the same as the one for mice tissue, with minor differences. The concentration of the blocking with $\mathrm{H}_{2} \mathrm{O}_{2}$ was increased up to $1 \%$. The blocking with $2,5 \%$ BSA was replaced by a blocking with $3 \%$ normal donkey serum (NDS), moreover, a concentration of $0,3 \%$ NDS was added to the antibody solutions. The following dilutions of the primary antibodies were used: 1:125 for rabbit anti-GENE-X, 1:50 for mouse anti-GFAP (Sigma Aldrich) and 1:50 for mouse antiNeuN (Millipore). Secondary antibodies (donkey anti-rabbit and donkey anti-mouse biotin labeled, Jackson ImmunoResearch) and the ABC kit was diluted 1:50.

Immunofluorescence stainings were carried out on human tissue, therefore it resembles the protocol for immunohistochemistry of human tissue. Two different conditions 
were used for the detection of the primary antibodies: one used fluorescent secondary antibodies and one used amplification of the signal with biotinilated secondary antibodies and fluorescent streptavidin. The secondary antibodies used were: donkey anti-rabbit Alexa Fluor 594 and donkey anti-mouse Alexa Fluor 488. (1:100, Jackson ImmunoResearch), donkey anti-rabbit- and donkey anti-mouse biotin labeled (1:100, Jackson ImmunoResearch). From this moment on, all steps were performed in the dark. For the amplification protocol, the slides with biotinylated secondary antibodies were incubated with streptavidin Alexa Fluor 594 (Vector Laboratories) diluted 1:250. Afterwards the tissues from all conditions were incubated with Hoechst (1:500). The slides were coverslipped with MOWIOL mounting medium.

To analyse tissues stained with $\mathrm{DAB}$, the Olympus $\mathrm{A} \times 70$ was used to perform transillumination microscopy in order to generate bright field images. The Olympus BX 51 was used to analyse the immunofluorescence stainings. By using the TXRED, FITC and DAPI filters, the Alexa 488, Alexa 594 and Hoechst probes could be visualized respectively.

\section{Results}

Human tissue
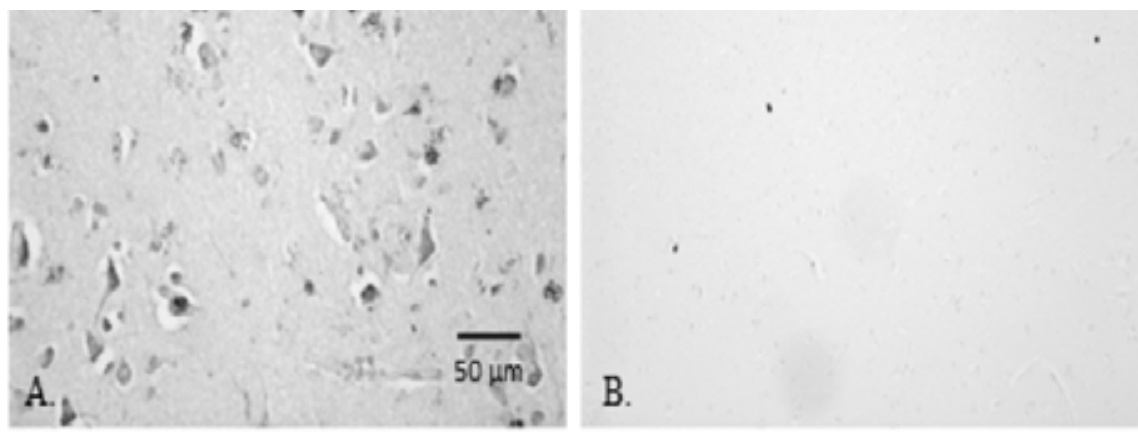

Figure 1. GENE-X expression in human neocortex. GENE-X expression was distinguishable in individual cells that, according to the pyramidal shape, seemed to be neurons (A). Neg. control without primary antibody was negative (B).

A pilot showed a retrieval step at $80^{\circ} \mathrm{C}$ in combination with a concentration of 1:125 of the primary antibody were the optimal conditions to stain GENE-X in human tissue. A clear expression of the GENE-X protein seemed to be visible in the soma (cytoplasm and nucleus) and in the proximal part of the axon of pyramid neurons, these were distinguished from 
glial cells based on their typical pyramidal shape (figure 1A). The protein was also present in the cytoplasm and nucleus of non-pyramidal cells. To prove the cell type, a double labelled staining with specific cell markers was required. The negative control slide without the primary antibody showed barely a staining signal (figure 1B).

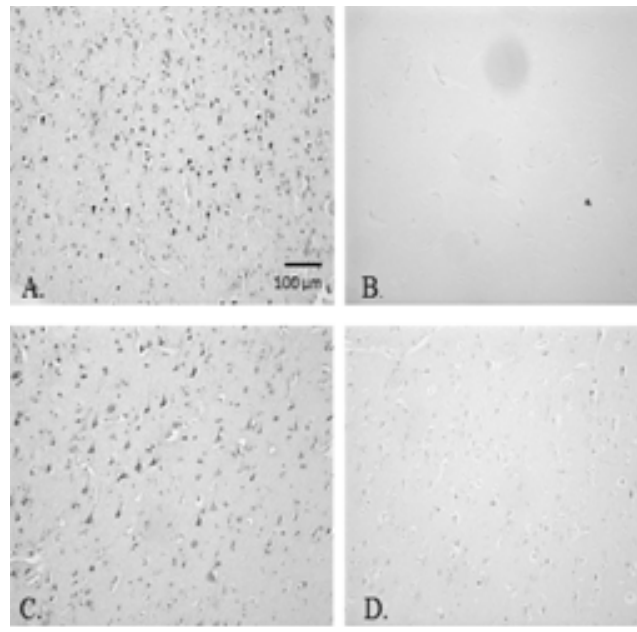

Figure 2. GENE-X expression in human neocortex with antigen as blocking peptide. $a: p=$ antibody:peptide ratio, Pos. control without blocking peptide as a baseline measurement $(A)$ and a neg. control staining without primary antibody $((a): p, 1: 10)$ to prove no direct interaction of blocking peptide with tissue (B). Signal intensity decreased when a: $p$ was increased ( $C(a: p=1: 2, D(a: p=1: 10)$.
To identify whether the binding of the GENE-X primary antibody was specific, the peptide used to synthesise this antibody was pre-incubated with the antibody itself prior to the staining. In this experiment the molar ratios antibody:peptide of 1:2, 1:5 and 1:10 were used. For each ratio a negative control was included. A positive control (figure $2 \mathrm{~A}$ ) gave the same results as before (figure 1A). Furthermore, when the antibody: peptide ratio increased, the intensity of the staining was reduced (figure $2 \mathrm{C}$ and $\mathrm{D}$ ). The negative controls of each condition tested were negative, representing there was no direct interference of the blocking peptide with the tissue.

In order to investigate the exact location of the expression of GENE-X in specific cell-types, a co-localization immunofluorescence staining was performed on GENE-X/NeuN and GENE-X/GFAP. First, a pilot proved the tissue to be applicable to stain GFAP and NeuN. A second pilot indicated that amplification of the GENE-X signal was preferred over amplification GFAP's or NeuN's signal (data of both pilots not shown). Unfortunately, the negative controls had a positive signal, regardless the secondary antibody used.

The co-localisation of GENE-X and GFAP implicated that the main expression of GENE-X (red) was not located in the astrocytes (green), since the green labels were barely merged with the red labels. However, the signal of GENE-X was for greater part merged with the signal of Hoechst (blue, label for nuclei). When all 3 signals were combined, it came clear 
that GENE-X was expressed in astrocytes to a low extent, and in nuclei of other cells. In the co-localization of GENEX and NeuN it appeared that these other cells were neurons, since the signal of GENE-X (red) and NeuN (green) were almost completely merged. Contractively, in this staining was barely a merge of GENE-X's signal and the signal of Hoechst, while the GFAP staining showed expression in nuclei. (No black and white data available)

\section{Mouse tissue}

A pilot showed a concentration of 1:250 of the primary antibody, together with preblocking by BSA are the best conditions to stain the mouse tissue. New pilot stainings had been performed on mouse tissue coming from two types of social defeat studies (pilot and batch). The staining of the pilot mouse model showed expression of GENE-X in cells in the PFC. The expression seemed to be increased in the PFC of the SD mice (figure $3 \mathrm{~A}$ ), when compared to the control mice (figure $3 \mathrm{~B}$ ). The pilot staining on the batch mouse model pointed out moderated results, since only a minimal expression of GENE-X was visible in the brain cells of both the SD and CTR tissue.
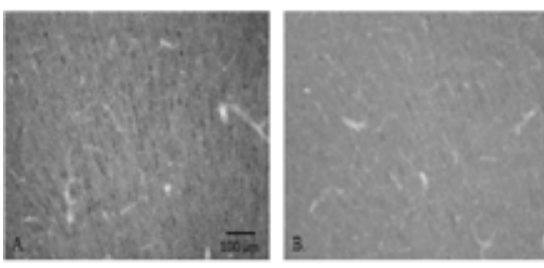

Figure 3. Expression of GENE-X in the prefrontal cortex (PFC) of social defeat (SD) and control (CTR) mice of the pilot mouse model. In the PFC the expression of GENE-X was increased in the SD mouse (A) when compared to the CTR mouse (B)
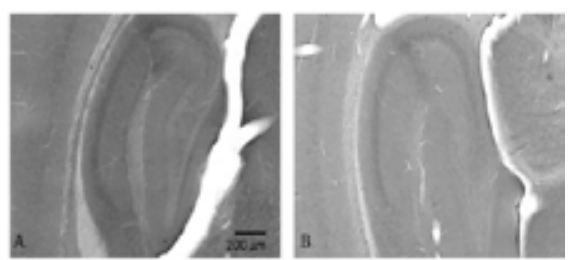

Figure 4. GENE-X expression in hippocampus of social defeat (SD) and control (CTR) mice of the batch mouse model. In the hippocampus the general expression of GENE-X was increased in the SD mouse

(A) when compared to the CTR mouse (B)

However, differences can be spotted in an image of the entire hippocampus (figure 4). These pictures were taken by using the same exposure time and light intensity to ensure the differences in grey value arose from differences in signal intensity. The expression of GENEX was abundant in the hippocampus of SD mice (figure $4 A$ ), compared to mice not exposed to stress (CTR) (figure $4 \mathrm{~B}$ ). However, both pilot stainins were carried out on several slides of only 1 animal for each condition. Based on these previous pilot results, an experimental staining on the tissue of the batch social defeat mouse model was performed. Two brains slides from 6 different social defeated animals and 6 controls were used. In this staining a negative control for each condition was included. After staining 
analysis, no GENE-X expression was visible in the neurons, neither in the glial cells, in both the SD tissue (figure $5 \mathrm{~A}$ ) and the CTR tissue (figure $5 \mathrm{~B}$ ).

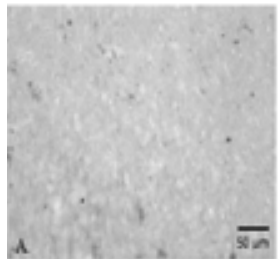

Figure 5. Experimental staining on GENE-X expression in social defeat (SD) and control (CTR) mice of the batch mouse model. Little or no expression in neurons nor glial cells, in neither the SD mice (A) and the CTR mice (B) tissue.
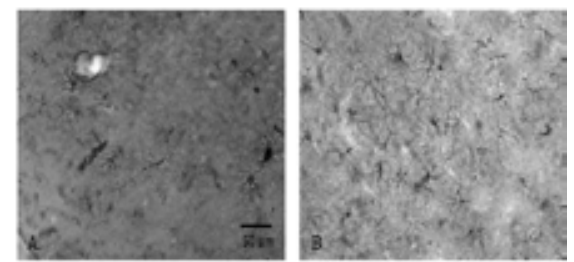

Figure 6. Expression of GFAP in social defeat (SD) (CTR) tissue of the batch mouse model, and on pos. control tissue. Expression of GFAP in SD (A) mice of the batch mouse model was not as abundant as in the pos. control (B). Background in the batch mouse model staining was severely increased compared to the positive control tissue.

To see whether these complications were caused by problems with the tissue or technical problems, a GFAP staining was executed on tissue of the batch mouse model. However, this time a positive control was included, this control consisted of tissue that previously had proven to be suitable to stain for GFAP. In the mouse model tissue, in both the SD and the CTR tissue, the expression of GFAP was very scarce, although in some places a couple astrocytes were visible (figure $6 \mathrm{~A}$ ). By contrast, the positive control tissue showed a clear expression of GFAP and the astrocytes are abundantly visible throughout the whole staining with less background staining (figure 6B).

\section{Discussion/Conclusion}

GENE- $X$ was expected to play a role in the susceptibility to develop PTSD after exposure to trauma. This pattern was seen in a previous research approaching soldiers who developed PTSD after deployment versus soldiers who were resilient to develop the disorder. This study showed hypomethylation of the promoter of GENE-X in relation to PTSD. Currently very little is known about the function of GENE- $X$ in the brain. So in order to prove a link between hypomethylation of GENE-X and susceptibility to develop PTSD, the aim of this research was to analyse the expression pattern of GENE-X in the human- and mouse brain. The results showed specific GENE-X expression in the human neocortex, since it was reduced when blocking with the immunizing peptide. The co-localisation study indicated GENE-X to be expressed in the neurons, and to a lower extend in astrocytes. According to problems with the tissue of the batch mouse model, no data have been found yet on the influence of stress (social defeat) and the expression of GENE-X. 
Staining of the human tissue argued that neither the secondary antibody nor the blocking peptide had a direct interaction with the tissue, since the negative and positive control conditions of the pilot staining were both negative. The staining that included the blocking peptide in combination with the blocking peptide, indicated that the GENE-X antibody used was highly specific. Pre-incubation of the antibody and the peptide allowed the antibody to bind to the peptides. When the concentration of peptide was in excess compared to the antibody, all antibodies were assured to be bound to the peptide and will not interfere with the tissue (2). As expected, increasing the ratio of peptide to antibody resulted in depletion of the signal (figure $2 \mathrm{C}$ and $\mathrm{D}$ )

Regarding the negative controls sections of the immunofluorescence pilot were not negative, autofluorescence of lipofuscin or flavoproteins might play a role. Autofluorescence was proven by examining a plain piece of tissue. The autofluorescence seen in the negative control was proportionally reduced when compared to the fluorescence signal derived from the positive conditions. This indicated that the signal of the double labelled staining was a merger of specific bound fluorescent probes and of autofluorescence. In subsequent stainings, Sudan Black would need to be used to diminish the autofluorescence (3).

According to the results of the double labelled immunofluorescence stainings, GENE-X was mainly expressed in the neurons. The signals of NeuN and GENE-X matched in almost every cell, despite the signal was not overlapping in the nucleus of the neurons as observed in immunostaining with DAB. This might be explained by competition of the NeuN and GENE-X primary antibodies in the nucleus of neurons. Regarding the results, the NeuN primary antibody had a stronger affinity towards the tissue and therefore intercepted the binding of the GENE-X antibody to its target protein in the nucleus. For this reason, GENE-X seemed to be expressed solely in the cytoplasm of neurons. The NeuN protein was expressed in the perinuclear cytoplasm too, although to a lower extend, therefore allowing the GENE-X antibody to interact with its target in the cytoplasm (4).

The co-localisation of GENE-X and GFAP showed a lower expression of GENE-X in astrocytes, primarily in the cytoplasm. In this staining, expression of GENE-X was present in the nucleus of cells other than astrocytes. Still, these cells were considered to be neurons, since the fusion of GENE-X and Hoechst was very frequent, and no other type of cells was that abundant expressed in brain tissue besides glial cells (4). 
Consequently, GENE-X was expressed in the nuclei of neurons and the cytoplasm of both neurons and astrocytes in the human neocortex. To counteract the competition of NeuN and GENE-X primary antibodies, a sequential double labelled staining should be performed. This would address the separate staining of NeuN and GENE-X in the same tissue.

The pilot staining in the tissue of the pilot mouse model showed encouraging results, GENE-X was expressed in various sites in the brain, amongst other things in the PFC and the hippocampus of both SD and CTR mice. Distinction could be made between these two conditions, since the SD tissue showed a more intense staining signal compared to the CTR (figure 3 and 4). The pilot on the batch mouse model displayed a lower general expression in both conditions, but the expression in the SD material was still higher compared to the CTR material. The increased expression in SD tissue in these 2 pilots was supposed to be caused by hypomethylation of the GENE-X promotor as a consequence of stress exposure, since the PFC and hippocampus are associated with stress, but an experimental staining on numerous tissue was needed to confirm.

The experimental staining was conducted on the material of the batch mouse model, regarding the pilot, the general expression was hypothesized to be low. Regrettably, no expression at all was detectable in any of the coupes (figure 5). Considering the pilot with GFAP antibody that followed (figure 6), this was probably caused by incorrect fixation of the tissue from the batch mouse model. Contrary to the material of the pilot mouse model that was perfused by fixative, the material of the batch mouse model was immerged fixed. Fixation by perfusion was preferred over immersion, since the fixative fluid would quickly reach every spot in the brain tissue through its natural vascular system, while during immersion not all the tissue would be fixed at the same time. Since immersion would take a considerable longer time, hypoxia could have started in the centre of the tissue before it was well prepared (5). On the other side, if the immerge fixation in formaldehyde was prolonged too long, the antigen could be masked by overflowing cross-linking and increased hydrophobicity of the tissue. In this case an antibody retrieval step or proteolytic digestion would be mandatory to break down the cross-links to render the reactivity of the antigen (5).

This research showed promising data on human material, but this was not in any way related to stress. The stainings on SD and CTR mouse tissue should have pointed out whether susceptibility to develop PTSD was correlated with GENE-X expression. However, 
technical problems with the tissue did not allow significant data to be acquired. To prove a causal link between increased expression of GENE-X due to hypomethylation of its promotor, further research would need to be conducted.

\section{Role of the student}

Renee Simons was an undergraduate student working under the supervision of Laurence de Nijs when the research in this report was performed. All stainings and processing of the data are performed by the student. The design of the research, together with the interpretation of the results were with guidance of the supervisor.

\section{References}

1. Zannas AS, Provençal N, Binder EB. Epigenetics of Posttraumatic Stress Disorder: Current Evidence, Challenges, and Future Directions. Biological Psychiatry.

2. Cahill CM, McClellan KA, Morinville A, Hoffert C, Hubatsch D, O’Donnell D, et al. Immunohistochemical distribution of delta opioid receptors in the rat central nervous system: Evidence for somatodendritic labeling and antigen-specific cellular compartmentalization. The Journal of Comparative Neurology. $2001 ; 440(1): 65-84$

3. Schnell SA, Staines WA, Wessendorf MW. Reduction of lipofuscin-like autofluorescence in fluorescently labeled tissue. Journal of Histochemistry \& Cytochemistry. 1999;47(6):719-30.

4. Herculano-Houzel S. The glia/neuron ratio: how it varies uniformly across brain structures and species and what that means for brain physiology and evolution. Glia. 2014;62(9):1377-91.

5. Werner M, Chott A, Fabiano A, Battifora $H$. Effect of formalin tissue fixation and processing on immunohistochemistry. Am J Surg Pathol. 2000;24(7):1016-9. 